\title{
Treatment Patterns Among Adults with Primary Immune Thrombocytopenia Diagnosed in Hematology Clinics in the United States
}

This article was published in the following Dove Press journal: Clinical Epidemiology

\author{
Leah J McGrath' \\ Karynsa Kilpatrick ${ }^{2}$ \\ Robert A Overman' \\ Diane Reams' \\ Anjali Sharma $\mathbb{D D}^{2}$ \\ Ivy Altomare ${ }^{3}$ \\ Jeffrey Wasser $\mathbb{D}^{4}$ \\ M Alan Brookhart ${ }^{1,5}$ \\ 'NoviSci, Inc., Durham, NC, USA; \\ ${ }^{2}$ Amgen Inc, Thousand Oaks, CA, USA; \\ ${ }^{3}$ Duke University Medical Center, \\ Durham, NC, USA; ${ }^{4}$ University of \\ Connecticut, Farmington, CT, USA; \\ ${ }^{5}$ Department of Population Health \\ Sciences, Duke University, Durham, \\ NC, USA
}

Correspondence: Leah J McGrath NoviSci, Inc, PMB 218, 201 W Main Street, Ste 200, Durham, NC 2770I, USA Tel + I 9193901642

Email Imcgrath@novisci.com
Purpose: Patients with immune thrombocytopenia (ITP) have low platelet counts and an increased risk of bleeding. We described treatment patterns and clinical outcomes in routine practice in the United States (US).

Patients and Methods: Using electronic health record data from hematology/oncology clinics linked to administrative claims in the US, we studied 447 adults newly diagnosed with primary ITP from 2011 to 2016. Patients with a secondary cause of thrombocytopenia were excluded. The incidence of ITP treatment initiation, bleeding events, and rescue therapy use were estimated using competing risk models.

Results: At 1-year post-ITP diagnosis, $50 \%$ of patients were prescribed an oral corticosteroid, with the majority being prescribed immediately following diagnosis. Of the more common secondline options, rituximab use was the most frequent (1-year cumulative incidence: 16\% [95\% confidence interval: 12,19$])$, followed by romiplostim $(9 \%[7,12]$ and eltrombopag $(5 \%[3,8])$. Use of these drugs was similar at 2 years post-diagnosis. At 6 months post-ITP treatment initiation, the cumulative incidence of bleeding was similar among eltrombopag and romiplostim initiators $(17 \%[6,33]$ and $19 \%[9,31]$, respectively) and was slightly lower in rituximab users $(12 \%[6,20])$. However, during this same timeframe, rituximab users had a higher incidence of rescue therapy use $(48 \%[36,58]$ versus $29 \%[14,46]$ in eltrombopag and $26 \%[14,39]$ in romiplostim users). Although splenectomy was rare, at 6 months post-surgery nearly $20 \%$ had experienced a bleed and nearly $20 \%$ had required rescue.

Conclusion: This study describes the health trajectory of adults with ITP who are managed in hematology clinics in the US and could inform the design of non-interventional studies of comparative effectiveness among treatments.

Keywords: primary immune thrombocytopenia, thrombopoietin receptor agonists, rituximab, splenectomy, real-world evidence

\section{Introduction}

Primary immune thrombocytopenia (ITP) is a rare acquired autoimmune disorder that affects approximately 3.3 per 100,000 adults per year. ${ }^{1}$ The disorder is characterized by low platelet counts and an increased tendency to bleed. Although ITP generally presents as a subtle-onset, chronic disorder in adults, clinical manifestations can range from petechiae, purpura, and bruising to overt bleeding such as gastrointestinal or intracranial hemorrhaging. ${ }^{2}$ When treatment is deemed necessary, typical first-line therapies include corticosteroids, intravenous immunoglobulin (IVIg), and $\mathrm{Rh}_{\mathrm{o}}$ (D) immune globulin, also referred to as anti-D immune 
globulin (IV anti-D). ${ }^{3-5}$ Relapse or failure to respond to these drugs may necessitate second-line treatment, which includes a variety of medical options and splenectomy.

Splenectomy has historically been considered the secondline therapy of choice but has declined in recent years, ${ }^{6,7}$ partially due to increased availability of medical options ${ }^{8}$ including the thrombopoietin-receptor agonists (TPO-RAs), eltrombopag and romiplostim. These drugs were initially approved in the United States (US) in 2008 for adults with chronic ITP (ITP > 12 months), who have had an insufficient response to previous ITP treatments. ${ }^{9,10}$ The label for romiplostim in the US has recently been revised to include all patients who have had an insufficient response to previous ITP treatments, regardless of ITP duration. Randomized controlled trials (RCTs) and recent clinical studies in splenectomized and non-splenectomized patients have provided evidence for the long-term efficacy and safety of these drugs. ${ }^{11-13}$ Additionally, fostamatinib, a spleen tyrosine kinase (Syk) inhibitor was approved in the US in April 2018 for the treatment of chronic ITP in adults who have had an insufficient response to prior therapy. ${ }^{14}$

Outside of Phase III trials for newer agents, there is limited evidence from RCTs to guide ITP treatment decisions. Additionally, some of the available medication options are not approved for use in ITP, but rather are used because of their efficacy in other autoimmune diseases or solid organ transplant immune suppression. ${ }^{15}$ As a result, the American Society of Hematology practice guidelines (2011) for ITP conclude that there is insufficient evidence to guide a sequence of treatment for patients who have recurrent or persistent thrombocytopenia with bleeding after first-line treatment; ${ }^{16}$ and the most recent International Consensus Report $(2010)^{17}$ indicates no preference for a particular second-line therapy. An update to the ASH guidelines recommends different treatments based on considerations of disease duration and patient preference (ie, shared decisionmaking), due to lack of published comparative studies including data on long-term outcomes. ${ }^{18}$ Decision-making in this setting is challenging and has even been described as controversial, ${ }^{19,20}$ likely leading to substantial variability in treatment patterns. Moreover, there have been few reports of ITP management in clinical practice and even fewer examining treatment based on duration of the ITP diagnosis. Therefore, we sought to provide a comprehensive description of ITP treatment patterns in clinical practice in the US. We also sought to describe the occurrence of clinically meaningful outcomes, including bleeding and rescue therapy use, after initiation of the more common second-line medical options.

\section{Materials and Methods Data Source}

Structured data from the Flatiron Health Electronic Record (EHR)-derived database (Flatiron Health, Inc.) were linked at the patient-level to the MarketScan ${ }^{\circledR}$ Commercial and Medicare Supplemental Databases (IBM Corp). The Flatiron EHR data system includes data from $>255$ hematology-oncology clinics, representing $>2330$ clinicians across the US. Records for diagnoses, laboratory results, and medications administered within the clinic are included in the database. The MarketScan databases capture healthcare claims for individuals covered by employersponsored private health insurance ( $<65$ years) and Medicare Supplemental insurance ( $\geq 65$ years). Available data include inpatient and outpatient diagnoses, procedures, and medications, which are identified via International Classification of Diseases, Clinical Modification, ninth or tenth revision (ICD-9-CM and ICD-10-CM), Healthcare Common Procedure Coding System (HCPCS), National Drug Codes (NDC), and/or Current Procedural Terminology (CPT) codes. Oral medications, including corticosteroids, were identified using the claims data, which captured all dispensed prescriptions regardless of care setting.

\section{Cohort Selection}

Patients were eligible for inclusion if they were aged 18 years or older at the time of a new ITP diagnosis (ICD-9: 287.31, ICD-10: D69.3) recorded in the EHR between January 1, 2011 and June 30, 2016. Patients were excluded if they had any diagnosis for a secondary cause of thrombocytopenia on or before the ITP diagnosis date (see eTable 1 for codes), received romiplostim or eltrombopag before the ITP diagnosis date, had evidence of a history of ITP documented in MarketScan, or no healthcare encounter in the 30 days following the diagnosis (to ensure they were "active" in the EHR). Patients were additionally required to have continuous enrollment in MarketScan in the twelve months before the ITP diagnosis to assess eligibility criteria and baseline characteristics (Figure 1).

\section{Outcomes and Follow-Up}

All outcomes were assessed using both Flatiron and MarketScan information; if there was a discrepancy between the date of the event between the two data sources, the earliest date was used. We assessed ITP treatments dispensed or administered following a diagnosis of ITP. ITP treatments were identified in the structured fields of the EHR or in the claims by ICD-9-CM, ICD-10-CM, HCPCS, NDC, DRG, and/or CPT codes. Treatment initiation was defined as the first dispensing for or administration of any of the following 
treatments: intravenous steroids, IVIg, IV anti-D, platelet transfusions, oral corticosteroids, splenectomy, azathioprine, alemtuzumab, cyclosporine A, cyclophosphamide, danazol, dapsone, eltrombopag, mycophenolate mofetil, rituximab, romiplostim, and vinca alkaloids. We considered oral corticosteroids, IVIg and IV anti-D as first-line medications, and all others as second-line/emergent medications.

Medical agents more commonly used in the second-line setting (eltrombopag, rituximab, and romiplostim) and splenectomy were of particular interest - we further subset our study population to patients initiating one of these therapies/procedures. Health events following a splenectomy or the initiation of one of the second-line therapeutic agents were defined as the first occurrence of the condition and identified using ICD-9-CM, ICD-10CM, HCPCS, NDC, DRG, and/or CPT codes in either the EHR or the medical claims (see eTable 2 for codes). We captured the following health events of interest: any bleeding event, intracranial hemorrhage, gastrointestinal hemorrhage, hematuria, ecchymosis, and epistaxis. We also identified the following medication events of interested: receipt of any ITP therapy (defined above), any rescue therapy, and receipt of the following individual treatments: oral corticosteroids, IV anti-D, IVIg, IV steroids, platelet transfusion, romiplostim, eltrombopag, rituximab, and splenectomy. We defined rescue therapy as receipt of IV anti-D, IVIg, IV steroids, or platelet transfusion.

Follow-up for ITP treatment initiation began 30 days after the date of ITP diagnosis, and for health events of interest began on the date of splenectomy or treatment initiation of commonly used second-line therapies. Patients were followed until the earliest occurrence of one of the following: 1) the outcome (eg, medications or health events of interest), 2) death, 3) end of data (December 31, 2016), or 4) end of health care, defined as loss of enrollment in MarketScan or 180 days without a clinic visit in Flatiron.

\section{Statistical Analysis}

Descriptive statistics were calculated for baseline covariates at the time of ITP diagnosis and re-calculated at receipt of splenectomy

\section{Cohort Entry Date \\ Diagnosis of ITP in EHR \\ Day 0}
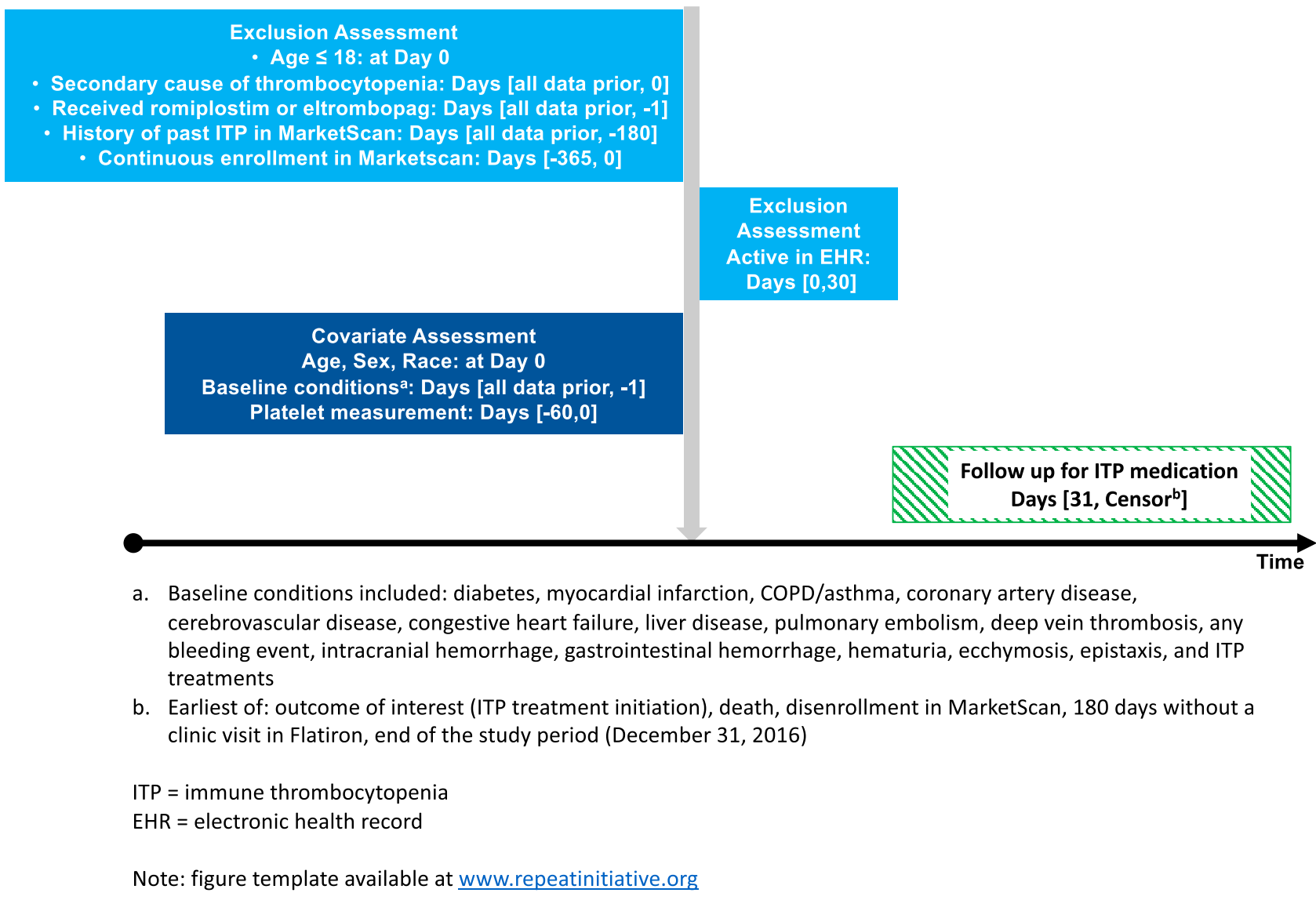

Figure I Study design schematic. 
or at the initiation of eltrombopag, rituximab, or romiplostim. The cumulative incidence of treatment initiation following an ITP diagnosis was measured for the entire cohort of patients with ITP. Estimates were provided specifically for 90 days, 1 year, and 2 years following diagnosis to describe treatment uptake in the newly diagnosed (ITP duration $<3$ months), persistent (ITP duration 3-12 months), and chronic (ITP duration >12 months) phases, respectively. ${ }^{21}$ Among the subset who initiated a secondline therapy, the cumulative incidences of the aforementioned health events of interest were quantified at 3 months and 6 months after splenectomy or initiation with the select medical therapies of interest. We used the non-parametric Aalen-Johansen estimator of cumulative incidence, ${ }^{22}$ and the associated $95 \%$ CIs, accounting for the competing risk of death. This analysis of secondary, deidentified data was approved by the Chesapeake Institutional Review Board. All analyses were conducted using $\mathrm{R}$, version $3.4 .0^{23}$ and the cmprsk package, version $2.2-7 .^{24}$

\section{Results}

\section{Study Cohort}

There were 4,552 patients with an ITP diagnosis in the EHR, and 447 patients met all eligibility criteria for inclusion (Figure 2). Of the included patients, 47\% were male, 61\% were white, and $32 \%$ were 65 years or older (Table 1). Prior to the ITP diagnosis, $52 \%$ had experienced any bleeding event, while $15 \%$ experienced a gastrointestinal hemorrhage specifically. Oral steroids were the most common treatment given prior to study entry (54\%); however, $42 \%$ of patients had received at least one rescue therapy. The median lowest platelet count in the 60 days prior to study entry was $85 \times 10^{9}$ / L (IQR: 39, 125).

\section{ITP Treatment Initiation}

\section{First-Line Medications}

Oral steroids were the most commonly used therapy after ITP diagnosis. The cumulative incidence of oral steroid use was 41\% (95\% confidence interval [CI]: 37, 46\%) at 90 days, $50 \%(46,55 \%)$ at one year, and $56 \%(51,61 \%)$ at two years (Figure 3, eTable 3). IVIg was used less often and most of the use occurred soon after ITP diagnosis (90-day, 1-year, and 2-year cumulative incidence: $11 \%$ [8, 14\%], 12\% [9, 15\%], and $14 \%[11,18 \%]$, respectively). IV anti-D use was rare (2-year cumulative incidence: $1 \%[0,2 \%]$ ).

\section{Second-Line/Emergent Treatments}

For treatments used beyond the first-line setting and/or in emergent settings, IV steroids were the most common. The 90day, 1-year, and 2-year cumulative incidences following ITP diagnosis were $16 \%$ [12, 19\%], 26\% [22, 30\%], and 34\% [29, $39 \%$ ], respectively (Figure 4, eTable 3). Of the treatments recommended in the second-line setting, rituximab was the most common, and use began soon after a diagnosis of ITP (90-day, 1-year, and 2-year cumulative incidence: 11\% [8, $14 \%$ ], 16\% [12, 19\%], and 18\% [14, 21\%], respectively) (Figure 5, eTable 3). Although TPO-RAs were used less

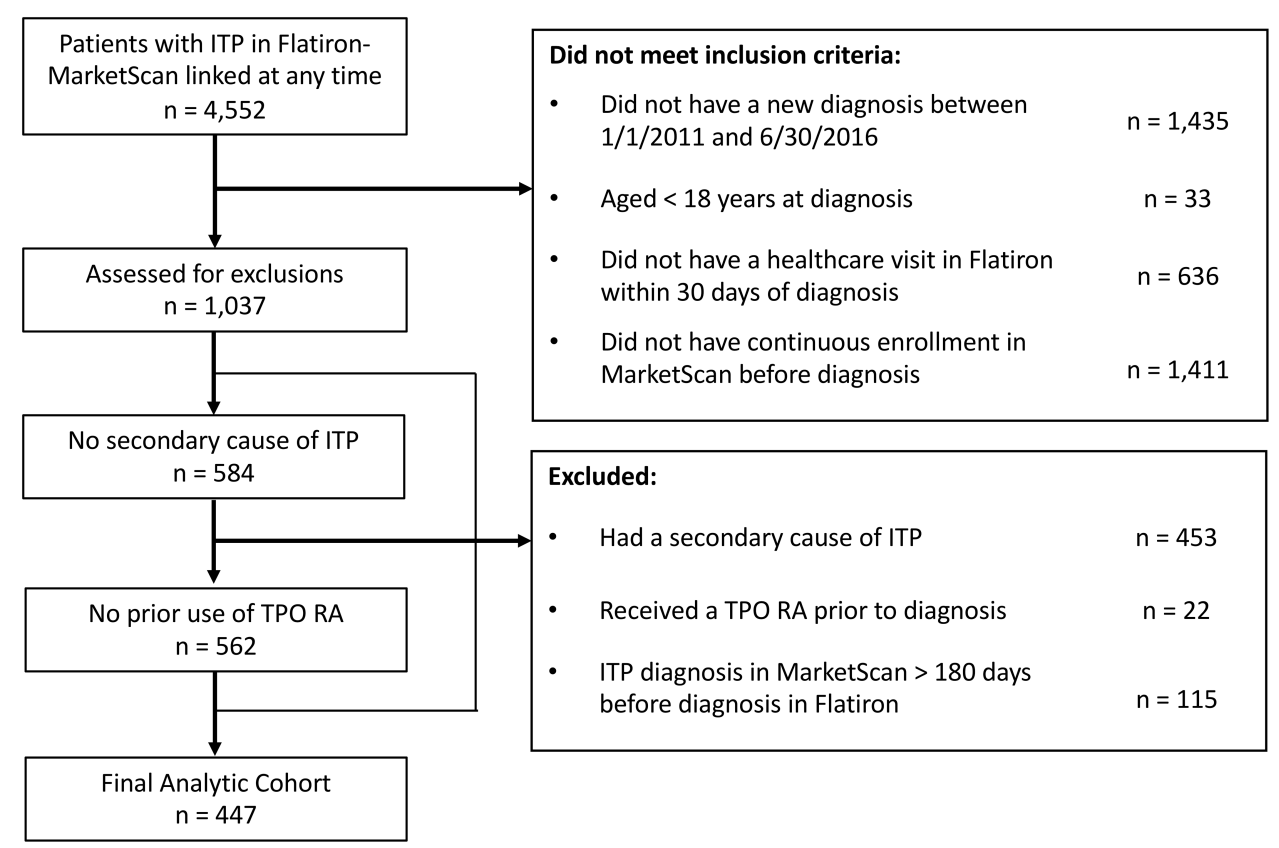

Figure 2 Study flow diagram. 
Table I Baseline Characteristics of Adults Diagnosed with Primary ITP, $201 \mathrm{I}-2016$

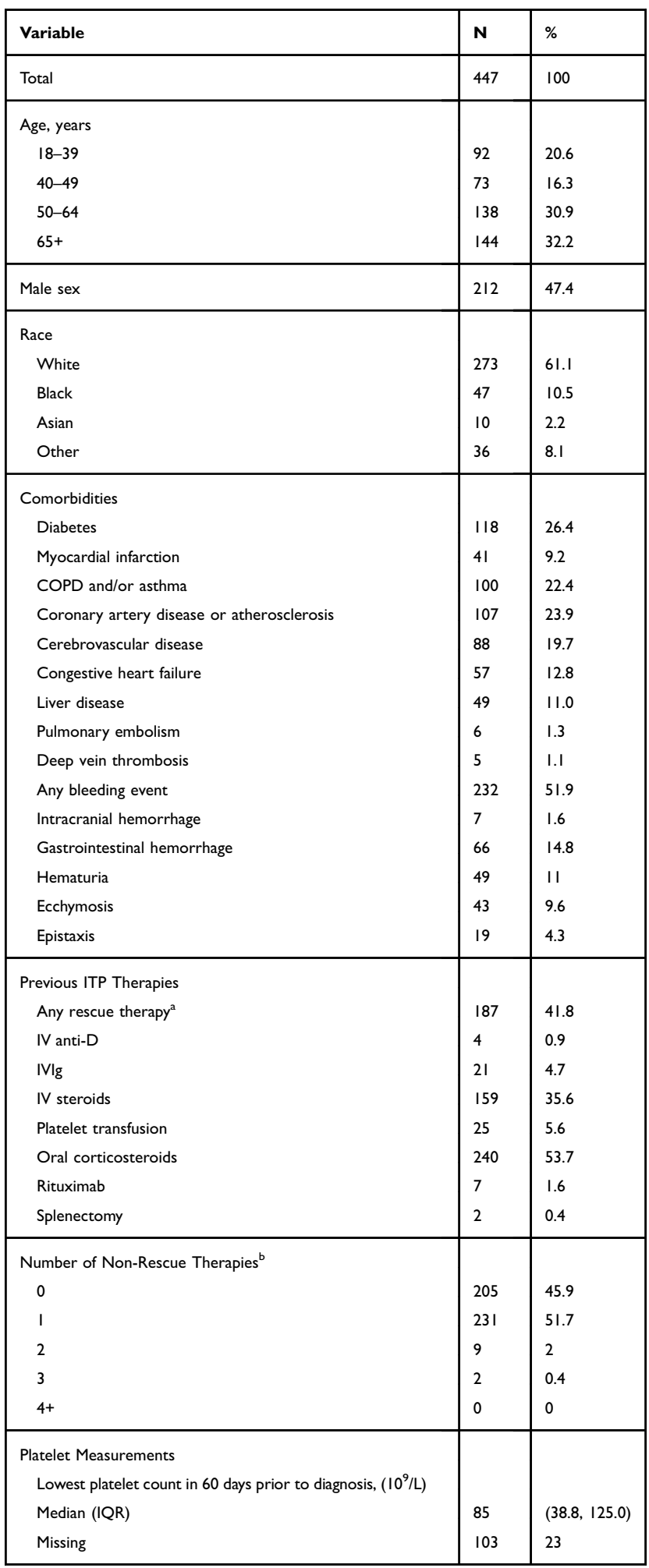

Notes: ${ }^{\text {aAny }}$ rescue therapy included IV anti-D; IVIg; IV steroids; platelet transfusion. bThere were no administrations of danazol, mycophenolate mofetil, vinca alkaloids (vincristine or vinblastine), or alemtuzumab. Less than $1 \%$ had a prior administration of azathioprine, cyclosporine A, cyclophosphamide, or dapsone.

Abbreviations: Anti-D, Rho(D) immune globulin; COPD, chronic obstructive pulmonary disease; IQR, interquartile range; ITP, immune thrombocytopenia; IV, intravenous; $\mathrm{IVIg}$, intravenous immunoglobulin.

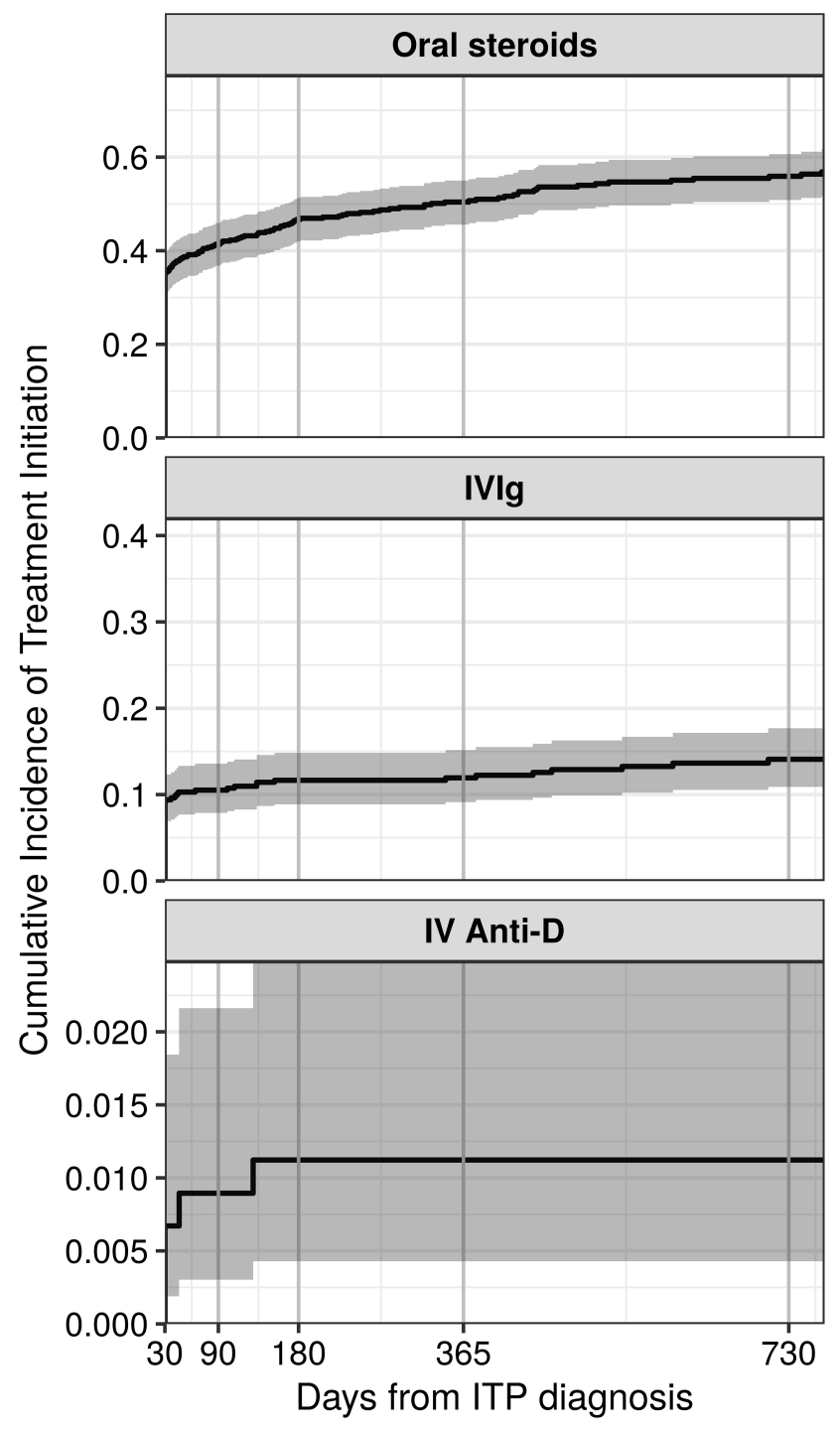

Figure 3 Cumulative incidence of therapies recommended in the first-line setting among patients with primary ITP in the United States, by time since ITP diagnosis.

frequently than rituximab, there was evidence of use before 12 months from the time of diagnosis, with $9 \%(7,12 \%)$ and $5 \%$ $(3,8 \%)$ of patients receiving romiplostim and eltrombopag within one year after diagnosis, respectively. Use of either drug after one year was minimal. Use of all other medical therapies was rare ( $<5 \%$ within 2 years, data not shown). Splenectomy was also infrequent (2-year cumulative incidence: $4 \%[2,6 \%]$ ), as was receipt of platelet transfusions (2-year cumulative incidence: 3\% [2, 5\%]) (Figure 4, eTable 3).

\section{Outcomes After Second-Line Therapy}

Following an ITP diagnosis, 21 patients underwent a splenectomy and 31,49 , and 84 patients initiated 


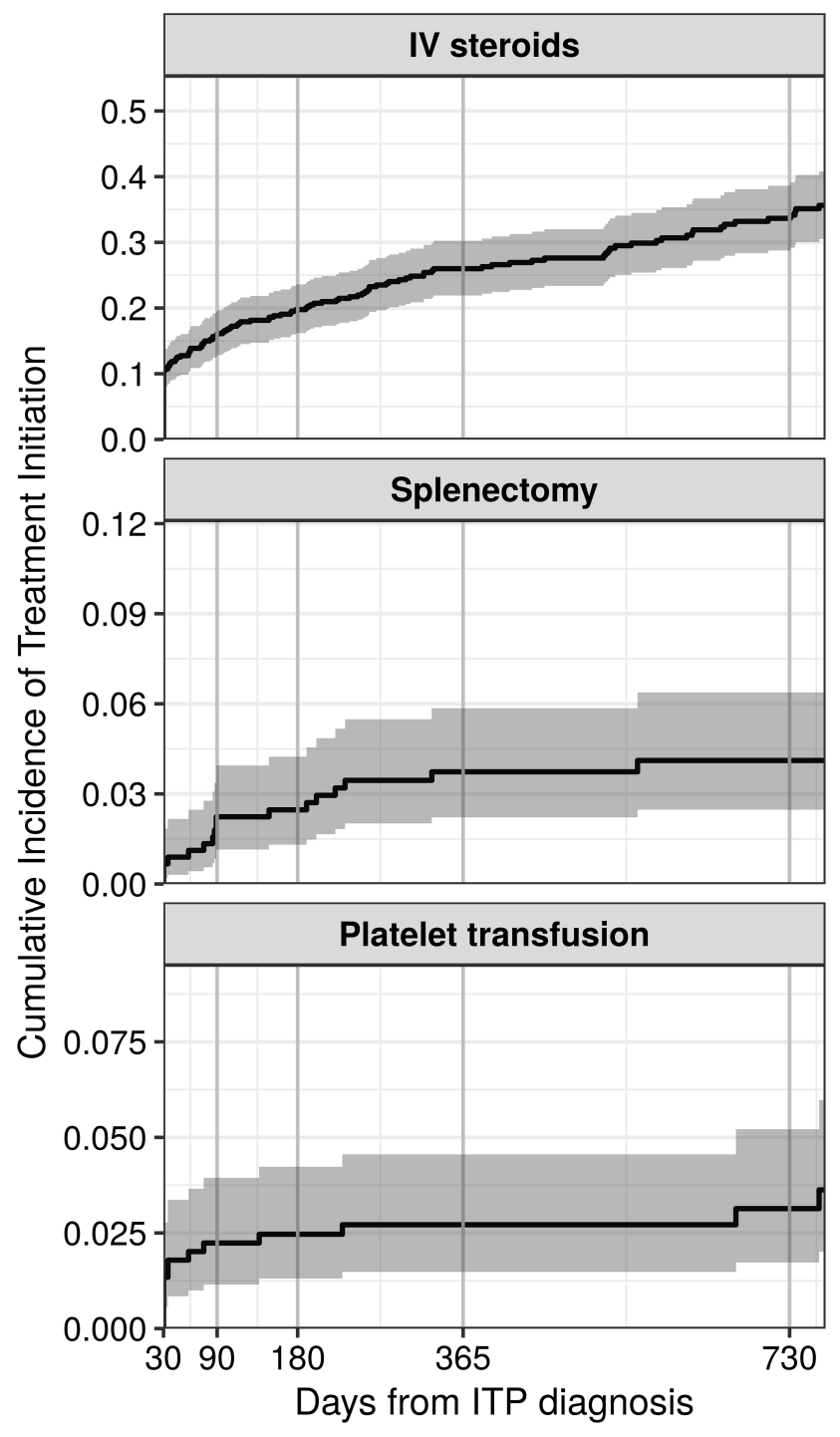

Figure 4 Cumulative incidence of therapies recommended outside of the first-line setting among patients with primary ITP in the United States, by time since ITP diagnosis.

eltrombopag, romiplostim, and rituximab, respectively, during follow-up (Table 2). The majority of patients receiving one of these treatments had prior bleeding, but this proportion was greatest in those who initiated one of the TPO-RAs (eltrombopag, 68\%; romiplostim, 65\%). Patients undergoing a splenectomy had a higher median platelet count in the 60 days prior to splenectomy compared to the medical treatment groups. Across treatment groups, nearly all had previous exposure to an oral steroid (86 - 100\%); prior IV steroid and IVIg use were also common.

Most of the bleeding events occurred in the first three months and at similar frequency following splenectomy (cumulative incidence: 14\% [95\% confidence interval (CI) 3 , 33\%])), rituximab $(11 \%[5,19 \%])$, and romiplostim $(15 \%[6,26 \%])$. (Table 3). However, the incidence of

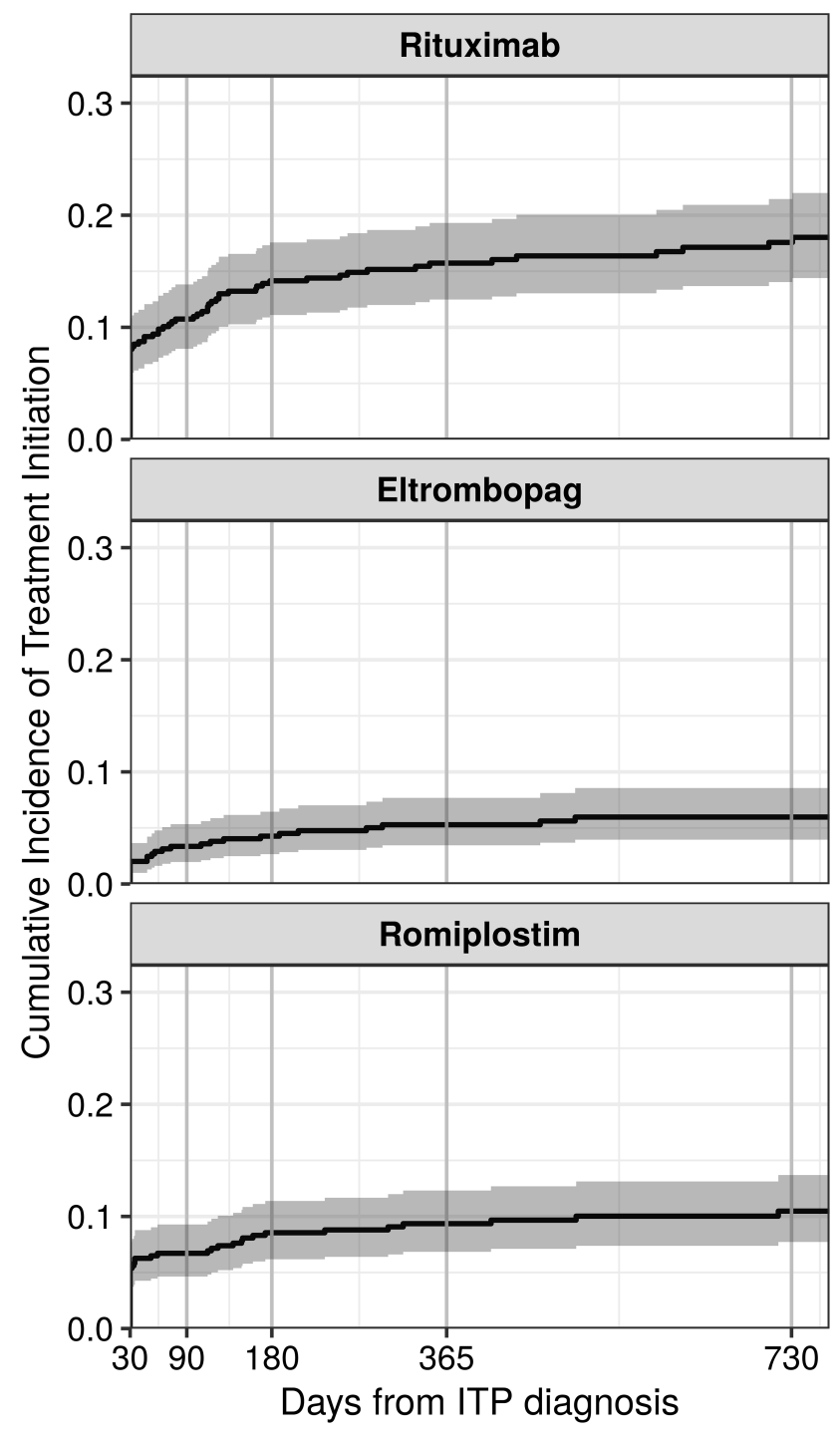

Figure 5 Cumulative incidence of common second-line treatment options among patients with primary ITP in the United States, by time since ITP diagnosis.

bleeding among patients initiating eltrombopag more than doubled from the 3- to 6-month timepoint after initiation. The 6-month incidence of bleeding in patients who initiated rituximab was slightly lower (12\% [CI: 6, 20\%]).

Rescue therapy use during this timeframe was highest in those who received rituximab. By 6 months following rituximab initiation, $48 \%$ had received a rescue therapy versus 29\% (CI: 14, 46\%) in eltrombopag users and 26\% (CI: 14, 39\%) in romiplostim patients. Rescue therapy use was lowest in the group who underwent splenectomy, [19\% (CI: 6, 39\%)], and most commonly occurred with administration of IV steroids and IVIg.

Oral steroid use outside of the rescue therapy setting was most frequent in the rituximab group (3-month cumulative incidence: $47 \%$ [CI: $36,57 \%]$ ), and lowest in the 
Table 2 Characteristics of Patients at Initiation of Select Second-Line Therapies

\begin{tabular}{|c|c|c|c|c|}
\hline Variable & Eltrombopag & Romiplostim & Rituximab & Splenectomy \\
\hline Total N & 31 & 49 & 84 & 21 \\
\hline Age, years, median (IQR) & $53(42,65)$ & $55(45,67)$ & $54(40,70)$ & $55(40,69)$ \\
\hline Male sex $(\%)$ & 42 & 47 & 37 & 38 \\
\hline White race (\%) & 71 & 69 & 61 & 71 \\
\hline \multicolumn{5}{|l|}{ Bleeding Events (\%) } \\
\hline Any bleeding event & 68 & 65 & 58 & 57 \\
\hline Intracranial hemorrhage & 0 & 4 & 1 & 10 \\
\hline Gastrointestinal hemorrhage & 19 & 25 & 12 & 14 \\
\hline Hematuria & 10 & 14 & 4 & 5 \\
\hline Ecchymosis & 16 & 14 & 20 & 14 \\
\hline \multicolumn{5}{|l|}{ Previous ITP Treatments (\%) } \\
\hline Oral steroids & 90 & 86 & 92 & 100 \\
\hline $\mathrm{IVIg}$ & 45 & 22 & 21 & 43 \\
\hline IV anti-D & 10 & 8 & 5 & 5 \\
\hline Eltrombopag & - & 8 & 6 & 14 \\
\hline Romiplostim & 32 & - & 6 & 29 \\
\hline Rituximab & 45 & 35 & $6^{\mathrm{a}}$ & 38 \\
\hline Splenectomy & 3 & 6 & 4 & - \\
\hline IV steroids & 55 & 51 & 44 & 67 \\
\hline Platelet transfusion & 3 & 8 & 2 & 5 \\
\hline \multicolumn{5}{|l|}{ Platelet Measurements } \\
\hline Lowest platelet count in 60 days prior $\left(10^{9} / \mathrm{L}\right)$, median (IQR) & $21(8,42)$ & $20(9,30)$ & $24(10,46)$ & $34(21,55)$ \\
\hline
\end{tabular}

Note: ${ }^{\text {aPrior }}$ use of rituximab among rituximab initiators occurred before ITP diagnosis.

Abbreviations: Anti-D, Rho(D) immune globulin; IQR, interquartile range; ITP, immune thrombocytopenia; IV, intravenous; IVlg, intravenous immunoglobulin.

eltrombopag group (3-month cumulative incidence: $7 \%$ [CI: 1, 19\%]) (Table 3). This suggests that rituximab patients were more likely to be on concomitant oral steroids at initiation or require oral steroids soon after rituximab was initiated. There was also evidence of switching between the TPO-RAs, with $23 \%(10,39 \%)$ of eltrombopag initiators receiving romiplostim and $15 \%(6,26 \%)$ of romiplostim initiators receiving eltrombopag within 6 months after initiation of their first TPO-RA (Table 3).

\section{Discussion}

We examined nearly 450 adults with newly diagnosed primary ITP managed in hematology clinics in the US. This cohort was a robust sample size for this rare disorder. Oral steroids were the most commonly used medication after diagnosis, reflecting their continued importance in the management of ITP. IV steroids were also common, with use steadily increasing by time since diagnosis. This suggests that IV steroids have a role in the initial management of patients and are likely utilized in emergent settings throughout the course of the disorder. However, the reasons for using specific rescue therapies were not available in the data. The most frequently used second-line medical options were rituximab, followed by romiplostim and eltrombopag, with use observed shortly after diagnosis for each.

We described clinical events of interest after initiation of second-line therapies, including bleeding, rescue therapy, and splenectomy. We focused on short-term risk of health events after the second-line options because splenectomy is a one-time surgical procedure, the median treatment duration for TPO-RAs in clinical practice has been shown to be 6 months, ${ }^{25}$ and the recommended treatment course for rituximab is a single weekly infusion for 4 weeks. ${ }^{16,17}$

The incidence of specific types of bleeding at 6 months after initiation of second-line therapies was rare, although patients on eltrombopag and romiplostim had a slightly higher incidence of gastrointestinal bleeding. It is possible this is due to a higher proportion of these patients with prior bleeding, including gastrointestinal bleeding, suggesting that these patients had more severe disease. The incidence of rescue therapy by 6 months was highest among patients receiving rituximab. This is not surprising 
Table 3 Cumulative Incidence (\%) of Events Following Initiation of Select Second-Line Therapies

\begin{tabular}{|c|c|c|c|c|c|c|c|c|}
\hline \multicolumn{9}{|c|}{ Percent ( $95 \%$ Confidence Interval) } \\
\hline \multirow[t]{2}{*}{ Event } & \multicolumn{2}{|c|}{ Eltrombopag } & \multicolumn{2}{|c|}{ Romiplostim } & \multicolumn{2}{|l|}{ Rituximab } & \multicolumn{2}{|c|}{ Splenectomy } \\
\hline & 3-Month & 6-Month & 3-Month & 6-Month & 3-Month & 6-Month & 3-Month & 6-Month \\
\hline Bleeding & & & & & & & & \\
\hline Any & $7(1,19)$ & $17(6,33)$ & $15(6,26)$ & $19(9,31)$ & II $(5,19)$ & $12(6,20)$ & $14(3,33)$ & $19(6,38)$ \\
\hline Ecchymosis & - & - & - & - & $2(0,8)$ & $2(0,8)$ & - & - \\
\hline Epistaxis & - & - & - & - & $I(0,6)$ & $I(0,6)$ & - & - \\
\hline Gl hemorrhage & - & $7(1,20)$ & $6(2,16)$ & $6(2,16)$ & $I(0,6)$ & $I(0,6)$ & - & - \\
\hline Hematuria & $3(0,15)$ & $3(0,15)$ & - & $2(0,10)$ & I $(0,6)$ & I $(0,6)$ & - & - \\
\hline Intracranial hemorrhage & - & - & - & - & I $(0,6)$ & I $(0,6)$ & $5(0,20)$ & $5(0,20)$ \\
\hline Subsequent Treatment & & & & & & & & \\
\hline Any rescue therapy ${ }^{\mathrm{a}}$ & $29(14,46)$ & $29(14,46)$ & $19(9,31)$ & $26(14,39)$ & $39(29,50)$ & $48(36,58)$ & $14(3,33)$ & $19(6,39)$ \\
\hline IV steroids & $16(6,31)$ & $16(6,31)$ & $13(5,24)$ & $19(9,32)$ & $36(26,46)$ & $4 \mid(31,52)$ & $14(3,33)$ & $14(3,33)$ \\
\hline IVlg & $23(10,39)$ & $23(10,39)$ & $8(3,18)$ & $8(3,18)$ & $10(4,17)$ & $15(8,24)$ & $5(0,20)$ & $10(2,27)$ \\
\hline Oral steroids & $7(1,19)$ & $10(2,24)$ & $29(17,43)$ & $34(21,48)$ & $47(36,57)$ & $53(42,64)$ & $38(18,58)$ & $48(25,67)$ \\
\hline Eltrombopag & - & - & $10(4,21)$ & $15(6,26)$ & $6(2,13)$ & $7(3,14)$ & $5(0,20)$ & $5(0,20)$ \\
\hline Romiplostim & $16(6,31)$ & $23(10,39)$ & - & - & $15(8,23)$ & $18(11,28)$ & $10(2,27)$ & $24(8,44)$ \\
\hline Rituximab & $13(4,27)$ & $13(4,27)$ & $10(4,21)$ & $15(6,26)$ & - & - & $10(2,27)$ & $14(3,33)$ \\
\hline Splenectomy & - & $7(I, 20)$ & $6(2,16)$ & $9(3,19)$ & I $(0,6)$ & $3(0,8)$ & - & - \\
\hline
\end{tabular}

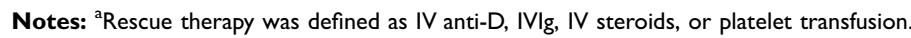

Abbreviations: $\mathrm{Gl}$, gastrointestinal; IV, intravenous; IVIg, intravenous immunoglobulin.

as previous studies have shown that fewer patients on rituximab achieved a platelet response compared with standard of care. ${ }^{26}$ Additionally, oral steroid use at 3 months was higher among patients initiating rituximab. It is possible that this could reflect combination therapy, as this treatment regimen has been proposed previously. ${ }^{27,28}$ Splenectomy rates were low and $19 \%$ of patients required further treatment within 6 months of splenectomy. The reasons for splenectomy failure are unable to be determined by our analysis. It is known that some patients do not respond to splenectomy, ${ }^{29-31}$ and reliable predictors of splenectomy response are lacking. It is possible that patients with more severe and/or refractory disease underwent splenectomy, therefore accounting for the $19 \%$ rate of rescue therapy so soon after the procedure.

Patterns of treatment observed in this study were generally similar to those seen in European studies. In a study of ITP patients being managed by hematologists in Germany, the most frequently used first-line therapies were steroids and IVIg. ${ }^{32}$ However, the relative use of rituximab and TPO-RAs in the second-line setting was lower $(10 \%$ and $6 \%$, respectively); and the uptake of splenectomy and other immunosuppressive agents was much higher than observed in our study ( $21 \%$ and $23 \%$, respectively). In a Spanish study of ITP patients in the hospital setting, steroids and immunoglobulins were the most common treatment modalities in the first-line setting. ${ }^{33}$ Of those requiring additional treatment, steroids, IVIg, and TPO-RAs were the most common choices. Similar to our results, many patients who initiated treatment with a TPO-RA did so soon after ITP diagnosis. Splenectomy was relatively rare $(6 \%)$; , and in contrast with our observations, rituximab use was also rare (1\%). Lastly, a chart review study from 5 European countries also found that while many treatments were used, corticosteroids were the most common (41\% of patients with chronic ITP used a corticosteroid over a 12 -month period), followed by IVIg; while just $6 \%$ underwent splenectomy. ${ }^{34}$

The limited number of studies in the US suggests similar treatment patterns among ITP patients being managed in routine clinical practice. In a survey of hematologist-oncologists in Oklahoma, nearly all (96\%) answered they would use corticosteroids in the first-line setting when treatment was indicated, with the remainder using IVIg. ${ }^{35}$ For patients who failed to respond with corticosteroids and IVIg, physicians reported that their next treatment option would include splenectomy (39\%), rituximab (37\%), and TPO-RA (16\%). In studies using administrative claims data in the US, rescue therapy use (IV steroids, IVIg, IV anti-D, and platelet transfusions) and receipt of splenectomy after ITP diagnosis have been examined. For 
commercially insured adults with ITP, 7\% of patients underwent splenectomy and 58\% received a rescue therapy, which were similar to our estimates. ${ }^{36}$ For elderly patients, data from Medicare demonstrated that 3\% of patients underwent splenectomy and 55\% received a rescue therapy after a diagnosis of ITP. ${ }^{37}$

Our study and previous research show that oral steroids are the most frequently used first-line treatment for the management of patients with primary ITP. Although oral steroids are the recommended treatment option in the first-line setting and in patients with symptomatic relapses in persistent or chronic ITP, the patient experience may be sub-optimal. In fact, we showed that almost all patients initiating one of the more common second-line therapies had a history of oral steroid use (range 86-100\%), although we were unable to discern the reason for the treatment switch (eg, treatment failure, side effects, etc.). Importantly, however, compared to patients who received rituximab or underwent splenectomy, we observed a lower incidence of oral steroid use after initiation of a TPO-RA. While the TPO-RAs were only approved for the treatment of chronic ITP during the time period studied for this analysis, ${ }^{9,10}$ we show that in practice, they are being administered shortly after diagnosis, perhaps as a method to decrease exposure to oral steroids.

In considering previous studies, our findings demonstrate the variability in the management of adults with primary ITP who require treatment beyond first-line therapy. This likely reflects the lack of prescriptive clinical guidance regarding the sequence or timing of treatment options in this setting ${ }^{18}$ and the uncertainty of who will respond to treatment. There are few studies that suggest that certain patient characteristics (eg, age, severity of thrombocytopenia) could be associated with response, but there is currently no biomarker available to predict individual response to treatment. Choice of therapy may also depend on lifestyle factors (eg, degree of daily physical activity), patient preference for surgery, and comorbidities that could limit certain types of therapy. A recent systematic review of studies evaluating the safety and efficacy/effectiveness of therapies used in the second-line setting discussed this issue. ${ }^{38}$ This review highlighted the lack of head-to-head RCTs directly comparing one treatment option to another and concluded that the strongest evidence for efficacy came from 10 placebo-controlled trials of TPO-RAs. These trials showed that patients on eltrombopag or romiplostim had higher rates of overall and complete platelet response and lower rates of rescue therapy and bleeding compared with placebo or standard of care treatment. ${ }^{38}$ Additionally, splenectomy was discussed as a standard treatment choice for chronic
ITP given the high likelihood of durable platelet response. Indeed, splenectomy is considered the treatment that results in the highest durable platelet response ${ }^{29}$ ranging from $66 \%$ to $74 \% .{ }^{39,40}$ Although we did not assess durable platelet response in our study, we did show that $19 \%$ of patients at 6 months required rescue therapy following splenectomy. Further research is needed to optimize medical treatment options in light of benefits and risks to standardize practice guidelines in this setting.

There are several limitations to consider when interpreting these results. The data from the EHRs arose primarily from community-based hematology clinics, and therefore may not be generalizable to other treatment settings such as academic treatment centers. A large number of patients were excluded because they did not have a clinic visit in the EHR data system within 30 days of the ITP diagnosis. This suggests that these patients were diagnosed in an alternate care setting prior to initiating care with the hematology specialist, such as a primary care provider. By excluding these patients, we ensured that the patients were actively contributing data to the EHR when they received their ITP diagnosis. We did not assess safety endpoints of interest, such as thrombotic events (eg, deep vein thrombosis) following initiation of ITP therapies. Of note, very few patients $(\sim 1 \%)$ had a history of deep vein thrombosis or pulmonary embolism, and these endpoints would likely be rare during follow-up. However, thrombotic events should be included in future safety studies. Additionally, we did not assess combination therapy (ie, rituximab plus oral steroids). It is possible that some of the oral steroid use observed soon after rituximab initiation was due to augmentation; however, this treatment pathway is not generally recommended due to a lack of long-term data. ${ }^{18}$ Finally, we used the date of ITP diagnosis that was recorded in the EHR; however, in some instances, there was evidence of prior ITP diagnoses reported in the administrative claims. Further investigation into the validity of the ITP diagnosis date in the EHR is warranted.

Our study is one of the first to describe patterns of treatment among newly diagnosed adults with primary ITP treated in hematology clinics in the US. Oral steroids continue to be used frequently to treat ITP, particularly soon after diagnosis. Administration of IV steroids was also observed often throughout all phases of the disorder. Medical therapies traditionally thought of as second-line options were also used early in the disease progression. Splenectomies were rare. Understanding current patterns of treatment and the incidence of subsequent clinical outcomes in patients with ITP could help inform the design of future non-interventional 
studies comparing effectiveness of various ITP treatments in terms of platelet response and bleeding outcomes.

\section{Data Sharing Statement}

Study data are available for purchase from Flatiron-Health, Inc.

\section{Acknowledgments}

This study was funded by Amgen Inc. This work was previously presented as an oral presentation entitled "Treatment Patterns Among Adults with Newly Diagnosed Primary Immune Thrombocytopenia in the United States" at the 2018 American Society of Hematology Conference. Robert A Overman's current affiliation and contact information is as follows: Blue Cross Blue Shield of North Carolina; robert. overman@bcbsnc.net.

\section{Disclosure}

$\mathrm{KK}$ and $\mathrm{AS}$ are employees of Amgen Inc and have stock ownership in Amgen Inc. LJM, DR, and MAB are employees and own equity in NoviSci, Inc. RAO was an employee at NoviSci, Inc. at the time the research was conducted. LJM reports grants from Amgen, Inc. MAB has had research support from Amgen and AstraZeneca, and has served as a scientific advisor/consultant to Amgen, Merck, TargetPharma, Genentech, AbbVie, and RxAnte. IA is a consultant to Amgen, Novartis, Genentech, Incyte, Bayer and an advisory board member of Celgene and Ipsen. IA reports personal fees from Amgen and Novartis. JW receives research funding from Incyte, Merck, Pfizer and Guardant, has stock ownership in Merck, Pfizer, Eli Lilly, Becton Dickinson, Abbott Labs, Biogen, and Allergan, and is a consultant to and reports personal fees from Amgen and Novartis. The authors report no other conflicts of interest in this work.

\section{References}

1. Terrell DR, Beebe LA, Vesely SK, Neas BR, Segal JB, George JN. The incidence of immune thrombocytopenic purpura in children and adults: a critical review of published reports. Am J Hematol. 2010;85 (3): 174-180.

2. Cines DB, Bussel JB. How I treat idiopathic thrombocytopenic purpura (ITP). Blood. 2005;106(7):2244-2251. doi:10.1182/blood-2004$12-4598$

3. Cooper N. Intravenous immunoglobulin and anti-RhD therapy in the management of immune thrombocytopenia. Hematol Oncol Clin North Am. 2009;23(6):1317-1327. doi:10.1016/j.hoc.2009.09.002

4. Kitchens CS, Pendergast JF. Human thrombocytopenia is associated with structural abnormalities of the endothelium that are ameliorated by glucocorticosteroid administration. Blood. 1986;67(1):203-206. doi:10.1182/blood.V67.1.203.203
5. Newland AC, Treleaven JG, Minchinton RM, Waters AH. High-dose intravenous $\operatorname{IgG}$ in adults with autoimmune thrombocytopenia. Lancet. 1983;1(8316):84-87. doi:10.1016/S0140-6736(83)91738-5

6. Cetin K, Wetten S, Christiansen C, Norgaard M, Heide-Jorgensen U. Recent time trends in the uptake of splenectomy in adults diagnosed with chronic immune thrombocytopenia: a nationwide historical cohort study in Denmark, 1996-2012. Haematologica. 2015;100 (Suppl 1):566.

7. Palandri F, Polverelli N, Sollazzo D, et al. Have splenectomy rate and main outcomes of ITP changed after the introduction of new treatments? A monocentric study in the outpatient setting during 35 years. Am J Hematol. 2016;91(4):E267-E272. doi:10.1002/ajh.24310

8. Lambert MP, Gernsheimer TB. Clinical updates in adult immune thrombocytopenia. Blood. 2017;129(21):2829-2835. doi:10.1182/ blood-2017-03-754119

9. PROMACTA Prescribing Information. 2018; Available from: https:// www.pharma.us.novartis.com/sites/www.pharma.us.novartis.com/ files/promacta.pdf. Accessed August 21, 2019.

10. Nplate Prescribing Information. 2018; Available from: https://www. pi.amgen.com/ /media/amgen/repositorysites/pi-amgen-com/nplate/ nplate_pi_hcp_english.pdf. Accessed August 21, 2019.

11. Bussel JB, Provan D, Shamsi T, et al. Effect of eltrombopag on platelet counts and bleeding during treatment of chronic idiopathic thrombocytopenic purpura: a randomised, double-blind, placebo-controlled trial. Lancet. 2009;373(9664):641-648. doi:10.1016/S0140-6736(09)60402-5

12. Kuter DJ, Bussel JB, Lyons RM, et al. Efficacy of romiplostim in patients with chronic immune thrombocytopenic purpura: a double-blind randomised controlled trial. Lancet. 2008;371 (9610):395-403. doi:10.1016/S0140-6736(08)60203-2

13. Bussel JB, Kuter DJ, Pullarkat V, Lyons RM, Guo M, Nichol JL. Safety and efficacy of long-term treatment with romiplostim in thrombocytopenic patients with chronic ITP. Blood. 2009;113 (10):2161-2171. doi:10.1182/blood-2008-04-150078

14. Bussel J, Arnold DM, Grossbard E, et al. Fostamatinib for the treatment of adult persistent and chronic immune thrombocytopenia: results of two Phase 3, randomized, placebo-controlled trials. Am J Hematol. 2018;93(7):921-930. doi:10.1002/ajh.25125

15. Provan D, Newland AC. Current management of primary immune thrombocytopenia. Adv Ther. 2015;32(10):875-887. doi:10.1007/ s12325-015-0251-z

16. Neunert C, Lim W, Crowther M, et al. The American Society of Hematology 2011 evidence-based practice guideline for immune thrombocytopenia. Blood. 2011;117(16):4190-4207. doi:10.1182/ blood-2010-08-302984

17. Provan D, Stasi R, Newland AC, et al. International consensus report on the investigation and management of primary immune thrombocytopenia. Blood. 2010;115(2):168-186. doi:10.1182/blood2009-06-225565

18. Neunert CE, Cooper N. Evidence-based management of immune thrombocytopenia: ASH guideline update. Hematol Am Soc Hematol Educ Program. 2018;2018(1):568-575. doi:10.1182/asheducation-2018.1.568

19. Lakshmanan S, Cuker A. Contemporary management of primary immune thrombocytopenia in adults. J Thromb Haemost. 2012;10 (10):1988-1998. doi:10.1111/j.1538-7836.2012.04876.x

20. Cuker A, Cines DB, Neunert CE. Controversies in the treatment of immune thrombocytopenia. Curr Opin Hematol. 2016;23 (5):479-485. doi:10.1097/MOH.0000000000000270

21. Rodeghiero F, Stasi R, Gernsheimer T, et al. Standardization of terminology, definitions and outcome criteria in immune thrombocytopenic purpura of adults and children: report from an international working group. Blood. 2009;113(11):2386-2393. doi:10.1182/blood2008-07-162503

22. Aalen O. Nonparametric estimation of partial transition probabilities in multiple decrement models. Ann Stat. 1978;6(3):534-545. doi:10.1214/aos/1176344198 
23. $R$ : a language and environment for statistical computing computer program. Vienna, Austria: R Foundation for Statistical Computing; 2017.

24. Cmprsk: subdistribution analysis of competing risks. $R$ package version 2.2-7. computer program; 2014.

25. McGrath L, Cetin K, Overman R, et al. Real-world patterns of romiplostim treatment in adults with primary immune thrombocytopenia in the United States. Am J Hematol. 2018;93(9):E45-E46. doi:10.1002/ajh.24965

26. Chugh S, Darvish-Kazem S, Lim W, et al. Rituximab plus standard of care for treatment of primary immune thrombocytopenia: a systematic review and meta-analysis. Lancet Haematol. 2015;2 (2):e75-e81. doi:10.1016/S2352-3026(15)00003-4

27. Gudbrandsdottir S, Birgens HS, Frederiksen H, et al. Rituximab and dexamethasone vs dexamethasone monotherapy in newly diagnosed patients with primary immune thrombocytopenia. Blood. 2013;121 (11):1976-1981. doi:10.1182/blood-2012-09-455691

28. Zaja F, Baccarani M, Mazza P, et al. Dexamethasone plus rituximab yields higher sustained response rates than dexamethasone monotherapy in adults with primary immune thrombocytopenia. Blood. 2010;115(14):2755-2762. doi:10.1182/blood-2009-07-229815

29. Chaturvedi S, Arnold DM, McCrae KR. Splenectomy for immune thrombocytopenia: down but not out. Blood. 2018;131 (11):1172-1182. doi:10.1182/blood-2017-09-742353

30. Navez J, Hubert C, Gigot JF, et al. Does the site of platelet sequestration predict the response to splenectomy in adult patients with immune thrombocytopenic purpura? Platelets. 2015;26(6):573-576. doi:10.3109/09537104.2014.959915

31. Ghanima W, Godeau B, Cines DB, Bussel JB. How I treat immune thrombocytopenia: the choice between splenectomy or a medical therapy as a second-line treatment. Blood. 2012;120(5):960-969. doi:10.1182/blood-2011-12-309153

32. Weide R, Feiten S, Friesenhahn V, et al. Outpatient management of patients with immune thrombocytopenia (ITP) by hematologists 1995-2014. Oncol Res Treat. 2016;39(1-2):41-44. doi:10.1159/000442769
33. Lozano ML, Revilla N, Gonzalez-Lopez TJ, et al. Real-life management of primary immune thrombocytopenia (ITP) in adult patients and adherence to practice guidelines. Ann Hematol. 2016;95 (7):1089-1098. doi:10.1007/s00277-016-2665-3

34. Rodeghiero F, Besalduch J, Michel M, Provan D, Grotzinger K, Thompson G. Treatment practices in adults with chronic immune thrombocytopenia - a European perspective. Eur J Haematol. 2010;84(2):160-168. doi:10.1111/j.1600-0609.2009.01361.x

35. Lu KH, George JN, Vesely SK, Terrell DR. Management of primary immune thrombocytopenia, 2012: a survey of oklahoma hematologists-oncologists. Am J Med Sci. 2014;347(3):190-194. doi:10.1097/MAJ.0b013e31827f4dd1

36. Altomare I, Cetin K, Wetten S, Wasser JS. Rate of bleeding-related episodes in adult patients with primary immune thrombocytopenia: a retrospective cohort study using a large administrative medical claims database in the US. Clin Epidemiol. 2016;8:231-239. doi:10.2147/CLEP.S105888

37. Li S, Molony JT, Cetin K, Wasser JS, Altomare I. Rate of bleeding-related episodes in elderly patients with primary immune thrombocytopenia: a retrospective cohort study. Curr Med Res Opin. 2017;1-8.

38. Bylsma LC, Fryzek JP, Cetin K, et al. Systematic literature review of treatments used for adult immune thrombocytopenia in the second-line setting. Am J Hematol. 2019;94(1):118-132. doi:10.1002/ajh.25301

39. Kojouri K, Vesely SK, Terrell DR, George JN. Splenectomy for adult patients with idiopathic thrombocytopenic purpura: a systematic review to assess long-term platelet count responses, prediction of response, and surgical complications. Blood. 2004;104(9):2623-2634. doi:10.1182/ blood-2004-03-1168

40. Kumar S, Diehn FE, Gertz MA, Tefferi A. Splenectomy for immune thrombocytopenic purpura: long-term results and treatment of postsplenectomy relapses. Ann Hematol. 2002;81(6):312-319. doi:10.1007/ s00277-002-0461-8
Clinical Epidemiology

\section{Publish your work in this journal}

Clinical Epidemiology is an international, peer-reviewed, open access, online journal focusing on disease and drug epidemiology, identification of risk factors and screening procedures to develop optimal preventative initiatives and programs. Specific topics include: diagnosis, prognosis, treatment, screening, prevention, risk factor modification,

Submit your manuscript here: https://www.dovepress.com/clinical-epidemiology-journal

\section{Dovepress}

systematic reviews, risk \& safety of medical interventions, epidemiology \& biostatistical methods, and evaluation of guidelines, translational medicine, health policies \& economic evaluations. The manuscript management system is completely online and includes a very quick and fair peer-review system, which is all easy to use. 